\title{
Perceived Health and Cause-Specific Mortality among Finnish Men and Women Aged 30 and over
}

\author{
PEKKA MARTIKAINEN, Ph.D., Researcher ${ }^{1}$ \\ ARPO AROMAA, D.Med.Sc., Research Professor ${ }^{2}$ \\ EERO LAHELMA, Ph.D., Professor ${ }^{3}$ \\ MARKKU HELIÖVAARA, D.Med.Sc., Chief Physician² \\ TIMO KLAUKKA, MD, Docent, Medical Research Officer ${ }^{4}$ \\ PAUL KNEKT, Ph.D., Head of Laboratory ${ }^{2}$ \\ JOUNI MAATELA, D.Med.Sc. ${ }^{5,6}$ \\ ANTTI REUNANEN, MD, Ph.D., Head of Laboratory ${ }^{2}$ \\ ${ }^{1}$ Population Research Unit, Department of Sociology University of Helsinki, Finland \\ ${ }^{2}$ National Public Health Institute (KTL), Helsinki, Finland \\ ${ }^{3}$ Department of Public Health, University of Helsinki, Finland \\ ${ }^{4}$ Social Insurance Institution (SII), Research and Development Center, Helsinki, Finland \\ ${ }^{5}$ Social Insurance Institution, Research and Development Center, Turku, Finland; ${ }^{6}(\dagger)$
}

\begin{abstract}
Perceived health is strongly associated with subsequent mortality, but the causes of this association are not known. The purpose of the paper is to analyze the association between perceived health and mortality after controlling for sociodemographic characteristics, and to estimate whether the association varies by cause of death. The study analyzed data from the Mini-Finland Health Survey, a nationally representative sample of 7,217 adults aged 30 and over in 1978-1980. A 12-year mortality follow-up was established by record linkage to death certificates at Statistics Finland. The study showed that perceived health was very strongly associated with mortality after sociodemographic adjustments; men reporting fairly poor or poor health had a 2.41 (95\% confidence interval 1.96-2.96) times higher adjusted mortality than men reporting fairly good or good health. Among women, the corresponding relative risk was 1.71 (95\% confidence interval 1.37-2.15). The strength of the association varied by cause of death. Future attempts to better understand the association between perceived health and mortality should begin at the cause-specific level. The explanations for the association are likely to vary for different diseases and causes of death.
\end{abstract}

Keywords: cause of death, mortality, perceived health 


\section{Introduction}

In health surveys and often in clinical studies, assessments of the level of general health and the presence of chronic conditions derive from personal interview information. In a measurement of perceived general health, the respondents are asked to describe their health on a scale ranging from good to poor. This global question correlates fairly well with other indices of health status, functional limitations, disability and assessments made by a physician (Johnson and Wolinsky 1993; Jylhä et al. 1986; Maddox and Douglas 1973; Tissue 1972). Perceived health is believed to summarize a broad range of medical and other health status information available to the individual, ranging from knowledge of existing diseases to cognitive and affective psychosocial characteristics of individuals and their personalities (Idler and Angel 1990; Tissue 1972).

In health research, the public health relevance of perceived health is partly based on its widespread use as an instrument in monitoring population health. Furthermore, perceived health has been found to predict health care use (Fylkesnes 1993; Hulka and Wheat 1985; Krakau 1991; Roos and Shapiro 1981) and mortality (Appels et al. 1996; Idler 1992; Idler, Kasl and Lemke 1990; Kaplan and Camacho 1983; Kaplan et al. 1996). To understand its nature, usefulness and limitations as a health measure, it is important to analyze its association with other health indicators, including mortality.

According to several studies, total mortality is much higher among persons reporting poor health than among those reporting good health (Appels et al. 1996; Idler 1992; Idler et al. 1990; Kaplan and Camacho 1983; Kaplan et al. 1996). Since both perceived health and mortality may be related to a wide range of sociodemographic and personal characteristics as well as chronic conditions, the association between perceived health and mortality could be mainly a reflection of such relationships. However, several previous studies suggest that this association remains after controlling for sociodemographic confounders, medically confirmed diagnoses and psychosocial factors (Idler and Benyamini 1997). A recent exception is a study by Kaplan and colleagues (Kaplan et al. 1996), who argue on the basis of data on middle-aged men in Eastern Finland that the association is to a great extent a reflection of underlying disease.

This paper attempts to shed more light on the association of perceived health and mortality by looking at two questions. First, we analyze the association of perceived health with subsequent mortality after controlling for sociodemographic characteristics. Second, we estimate whether the association varies by cause of death. 


\section{Material and Methods}

\section{Population sample}

A national sample of 8,000 adults was studied in the Mini-Finland Health Survey carried out in 1978-1980; altogether 3,637 men and 4,363 women. The two-stage random sample was drawn from population registers to represent Finnish adults aged 30 and over. The subjects were invited to attend a personal health interview and a two-phase health examination (Aromaa et al. 1989). Altogether, 7,703 persons $(96 \%)$ were interviewed by public health nurses in their homes or in the place where they were under permanent care. The Social Insurance Institution's Mobile Clinic Unit carried out the first phase of a two-phase health examination 1-6 weeks after the personal interview, and 7,217 persons (90\%) participated. In the second phase, further examinations were carried out on those with histories of major chronic conditions or symptoms or signs suggestive of such conditions. The current analyses are based on the 7,217 persons attending the first phase of the health examination.

\section{Measurement}

The baseline personal interview included questions about sociodemographic characteristics, chronic conditions, disability and various other measures of health status. The invitation to the health examination included a self-administered questionnaire, which provided further information on e.g. health status, chronic conditions and their treatment, job and job characteristics. At the beginning of the health examination the nurses checked the questionnaires, which had been filled out at home. Further interviews, tests and examinations followed and have been described more fully elsewhere. (Aromaa et al. 1989; Heliövaara et al. 1993.)

The questionnaire that was filled out before the medical examination included a question about perceived health. The question read: "How would you assess your current health?" The response alternatives were good, fairly good, intermediate, fairly poor and poor. The test-retest reliability of perceived health has been shown to be fair or good for both men and women (Lundberg and Manderbacka 1996; Martikainen et al. 1998). Reliability analyses from this data show that unweighed agreement of the 'good-intermediate-poor' categorization of perceived health was around $70 \%$ and unweighed Kappa values were around 0.5. Only among the over 74-year-olds did reliability decline below these levels.

Age at interview was classified into 5-year age groups: $30-34,35-39, \ldots, 75-79$, and $80+$. Marital status was classified into five categories: married, single, cohabiting, divorced, and widowed. Five ordinal educational categories were distinguished on the basis of the highest completed certificate or degree. These were primary, basic, lower intermediate, higher intermediate and higher education. The 
regional classification was based on the five social insurance regions: North, West, East, Southwest and South of Finland. Furthermore, we distinguished between the institutionalized and the noninstitutionalized population.

\section{Mortality and causes of death}

Information on date of death and cause of death was obtained from the national registers on population and cause of death by record linkage using the personal identification number. Mortality follow-up was for 12 years from recruitment. Altogether, the study population lived 78,762 person-years during follow-up $(35,315$ person-years among men and 43,447 among women). Classification of cause of death was based on the three-digit codes of the Finnish version of the International Classification of Diseases (ICD 8) (Lääkintöhallitus 1969). For the purposes of this study, the causes were grouped into broad categories as follows: All causes, malignant neoplasms (ICD 140-209), diseases of the circulatory organs (ICD 390459), all other diseases, and accidents and violence (E800-E999). 761 and 630 deaths occurred among men and women respectively.

\section{Missing data}

Missing data was observed for three of the explanatory variables studied. Those with missing information were classified into the largest single category of that variable. Only $0.1 \%$ of subjects had missing information for perceived health in the questionnaire. An equally small proportion had missing data on marital status, and information on education was missing for 20 subjects $(0.3 \%)$

\section{Statistical methods}

The analysis of the association between perceived health and mortality was carried out by means of Cox proportional hazards models (Clayton and Hills 1993). The person-years at risk were calculated on a daily basis. The STATA (Stata Reference Manual 1992) statistical package was used in fitting the models and performing likelihood-ratio tests between two models.

The results of the Cox models were presented as relative mortality risks (hazard ratios). The first category of each explanatory variable was the reference group, with a relative risk of one. We added sociodemographic background factors to the regression model in the time order that their values are most likely to have been determined (i.e. age first and institutionalization last). 


\section{Results}

\section{Perceived health and total mortality by duration of follow-up}

Table 1 presents person-years, number of deaths and relative mortality risks for the variables used in these analyses. Age-adjusted mortality for men reporting intermediate health was $42 \%$ (relative risk $1.42,95 \%$ confidence interval $1.17-1.72$ ) higher than for men reporting good or fairly good health in the 12-year follow-up period (Table 2). Similarly, men reporting fairly poor or poor health had $153 \%$ higher age-adjusted mortality. Controlling for education, marital status, region of residence and institutionalization had little bearing on these relative risks: after all adjustments, excess mortality was 39\% among men reporting intermediate health and $141 \%$ among men reporting fairly poor or poor health.

The association between perceived health and mortality was similar among both women and men. Among women, however, the association was somewhat weaker. Age-adjusted mortality for women reporting intermediate health was $36 \%$ higher than for women reporting good or fairly good health. Those women reporting fairly poor or poor health had $84 \%$ higher age-adjusted mortality. Among women as among men, controlling for other potential sociodemographic confounders than age had only a very limited impact on the association.

\section{Perceived health and mortality by cause of death}

The strength of the association between perceived health and mortality differed by cause of death (Table 3). Among men, the association was most marked for the group of other diseases (diseases other than malignant neoplasms and circulatory diseases): mortality was $102 \%$ and $326 \%$ higher for those reporting intermediate and fairly poor or poor health than it was for those reporting good or fairly good health respectively. The association was somewhat weaker for accidents and violence (49 deaths) and for circulatory diseases (about the same level as all causes of death combined). The smallest excess mortality was observed for malignant neoplasms. The excess cancer mortality of men reporting fairly poor or poor health was $64 \%$. The ranking of causes of death was similar when looking at the excess mortality of men reporting intermediate as well as fairly poor or poor health.

Judging by the excess mortality of women reporting fairly poor or poor health, the association between perceived health and mortality was largest for other diseases and circulatory diseases. Differentials were smaller for malignant neoplasms and accidents and violence. Overall, the pattern of cause-specific excess mortality by perceived health was similar, but the association was weaker among women than men. However, women reporting intermediate health had excess mortality of around $45-50 \%$ for all causes except malignant neoplasms; for malignant neoplasms the 


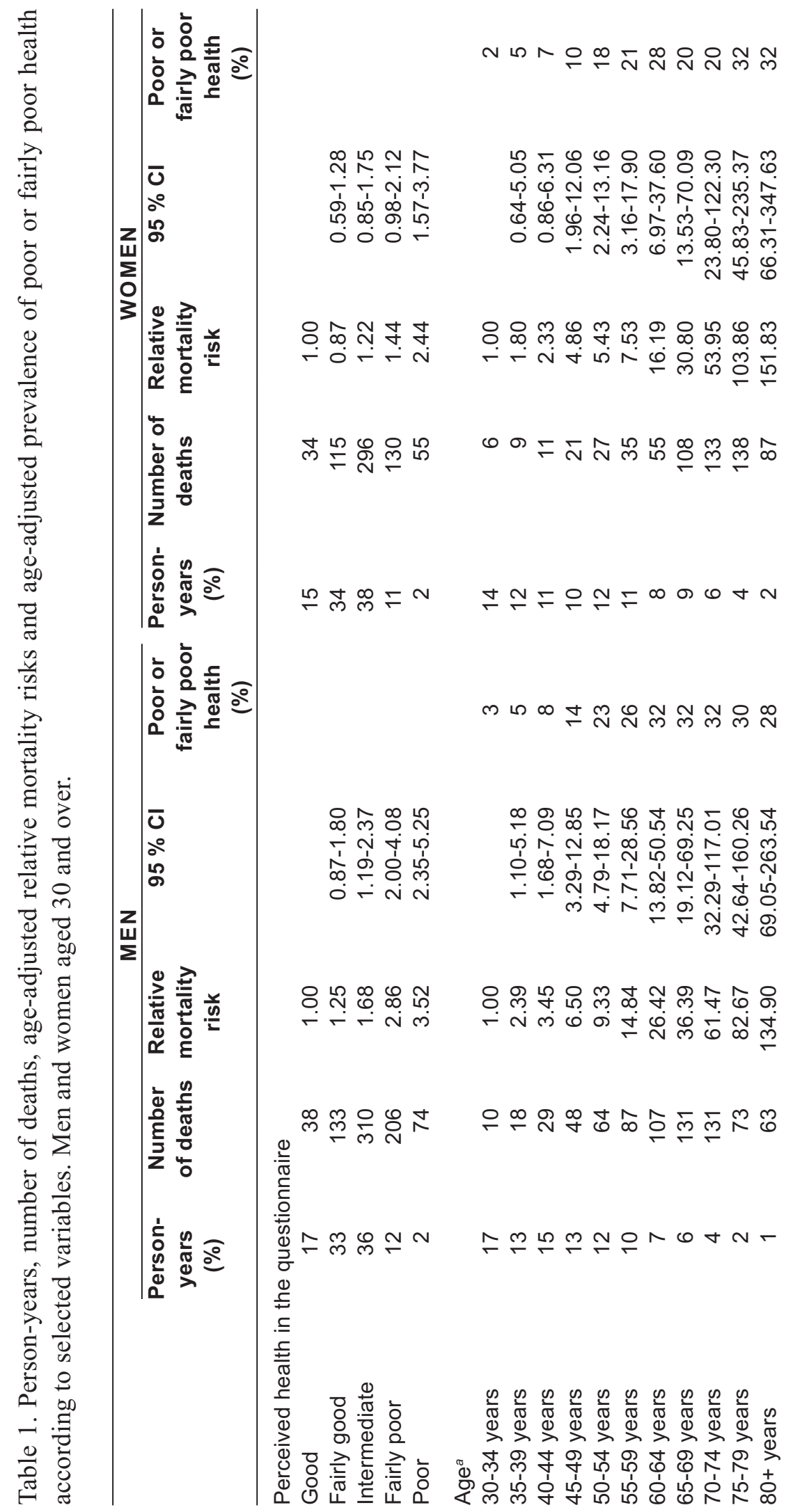




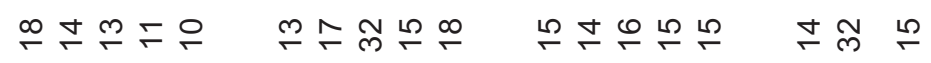

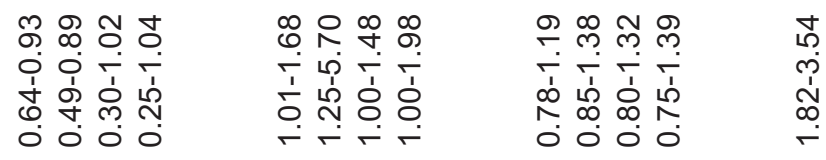

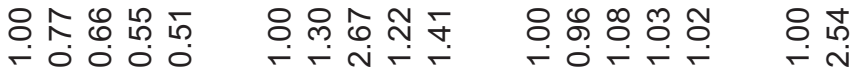

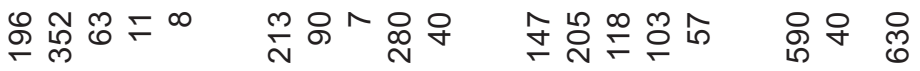

の ํํㅇ

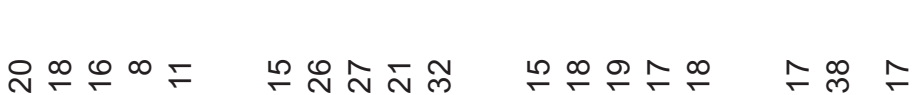

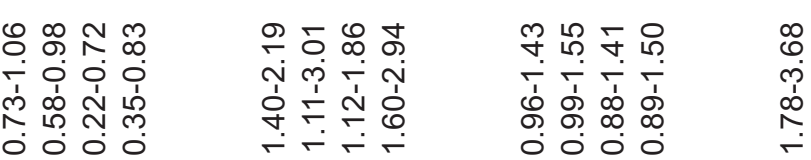

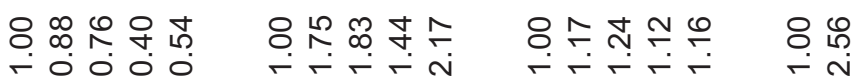

ㄸํㅇㅇㅁㄴㅠ 뀽요

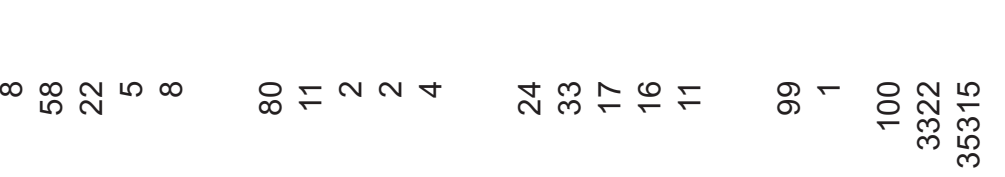

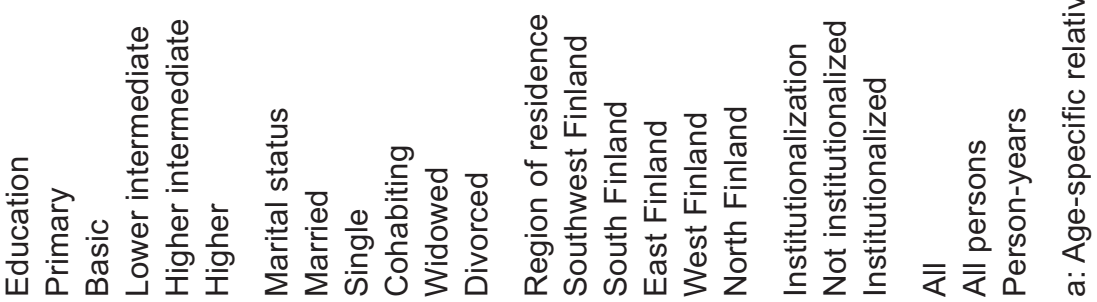


excess was only $10 \%$. Adjusting for sociodemographic variables had little effect on the cause-specific relative risks.

Table 2 . Relative mortality risks by perceived health $(1.00=$ those reporting good or fairly good health) according to six cumulative models, and $95 \%$ confidence intervals (CI). Men and women aged 30 and over.

\begin{tabular}{|c|c|c|c|c|c|c|}
\hline & Model & & & & & \\
\hline $\begin{array}{l}\text { Perceived } \\
\text { health }\end{array}$ & $\begin{array}{l}\text { Perceived } \\
\text { health }\end{array}$ & +Age & +Education & $\begin{array}{c}\text { +Marital } \\
\text { status }\end{array}$ & $\begin{array}{c}\text { +Region } \\
\text { of } \\
\text { residence }\end{array}$ & $\begin{array}{c}\text { +Institutionali- } \\
\text { zation } \\
(95 \% \mathrm{Cl})\end{array}$ \\
\hline
\end{tabular}

Men

$\begin{array}{lrrrrrr}\text { Intermediate } & 2.57 & 1.42 & 1.37 & 1.38 & 1.40 & 1.39(1.15-1.69) \\ & & & & & & \\ \text { Poor or fairly poor } & 5.79 & 2.53 & 2.41 & 2.45 & 2.45 & 2.41(1.96-2.96)\end{array}$

Women

\begin{tabular}{lrrrrrr} 
Intermediate & 2.59 & 1.36 & 1.31 & 1.31 & 1.31 & $1.32(1.08-1.62)$ \\
Poor or fairly poor & 4.62 & 1.84 & 1.74 & 1.74 & 1.74 & $1.71(1.37-2.15)$ \\
\hline
\end{tabular}

\section{Discussion}

\section{Summary of the results}

This study showed that perceived health is very strongly associated with subsequent mortality. In accordance with most other studies, the association existed even after controlling for age, educational attainment, marital status, region of residence and institutionalization.

The association between perceived health and total mortality was somewhat stronger among men than women: the adjusted relative risk of those reporting fairly poor or poor health as opposed to those reporting good or fairly good health was 2.41 among men and 1.71 among women. This sex difference was not due to a difference in the distribution of perceived health - among both men and women, about $50 \%$ had good or fairly good health and slightly less than $15 \%$ had fairly poor or poor health - but it may reflect the more severe nature of the underlying health problems that contribute to men's health perceptions. The strength of the association varied by cause of death. 


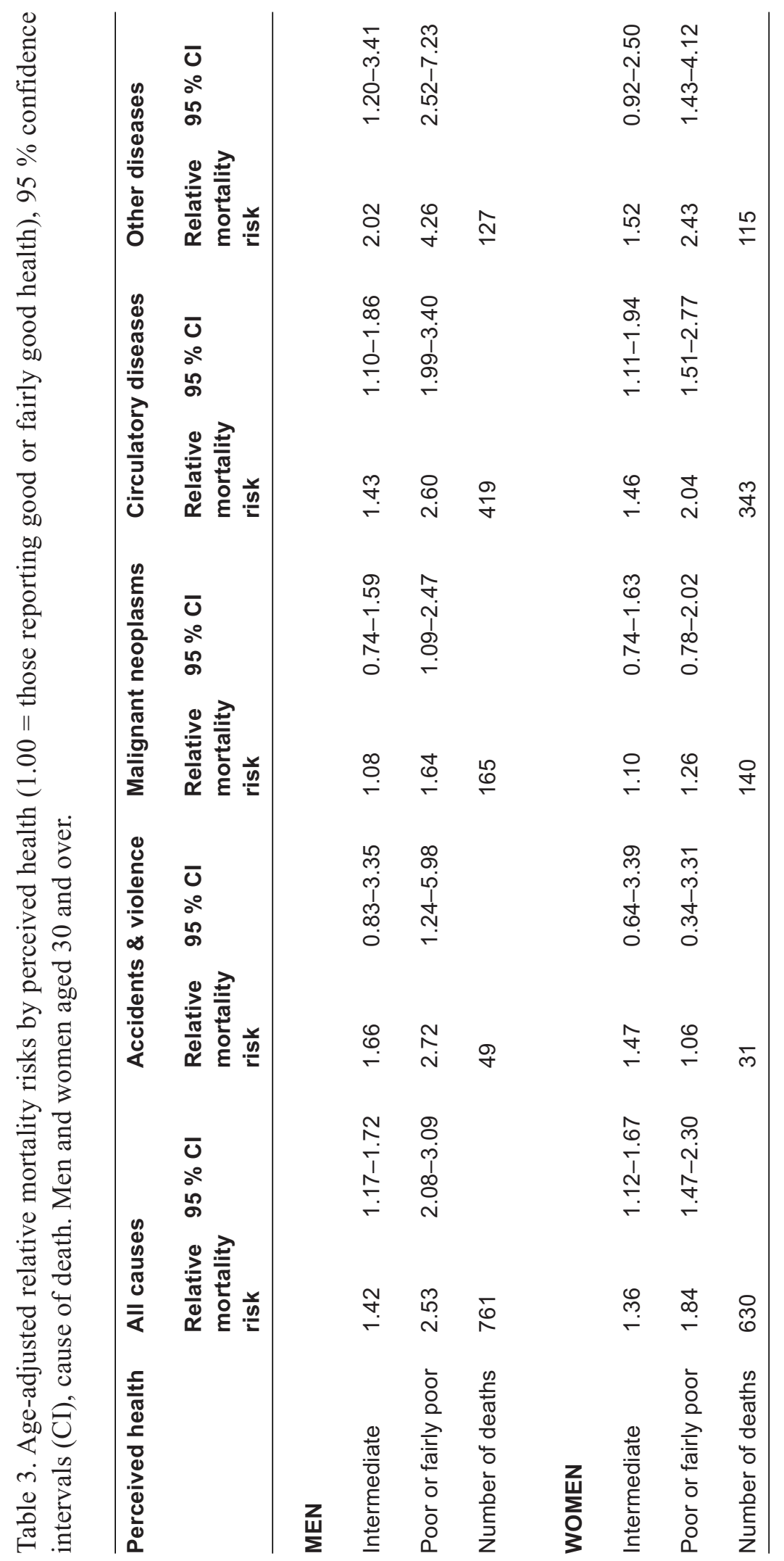




\section{Explanations and mechanisms}

The following main explanations for the association between perceived health and mortality can be proposed: (1) perceived health and mortality are related because both are affected by the same sociodemographic characteristics, pre-existing physical health, risk factors or other covariates, (2) perceived health mediates the effects of psychosocial characteristics on mortality, (3) perceived health has an independent effect on mortality. The mechanisms of the possible independent effects of perceived health on mortality are largely unknown. They may be related, for example, to immune 'suppression' and to the functioning of the immune system in general (Rakowski, Mor and Hiris 1991) or are associated with negative self-images that lead to passivity, delay and neglect in protecting one's health (Appels et al. 1996).

It is difficult to disentangle the different explanatory models and mechanisms from the research available so far. Controlling for as many health-related covariates as possible is not necessarily of great explanatory value, especially when the different covariates have been measured - often inadequately - at one point in time, and the causal and temporal relationships between perceived health and these covariates are often unknown. Only when longitudinal data on perceived health and measures of clinical disease and ill-health are available can we discriminate whether the association between perceived health and mortality is due to confounding, independent causation or mediation. However, some tentative observations and guidelines for further research can be made on the basis of the results presented here.

Our study suggests that adjustment for sociodemographic factors is of little relevance; after age is controlled for, education, marital status, region of residence and institutionalization have a relatively minor effect on the excess mortality of those reporting their perceived health below good.

If the association between perceived health and mortality is mainly due to confounding by pre-existing ill-health and known risk-factors, as suggested by Kaplan and colleagues (Kaplan et al. 1996), the association may be assumed to be strongest for chronic diseases. The relatively weak association between perceived health and malignant neoplasm mortality does not, however, support this view. On the other hand, the association between perceived health and circulatory disease mortality was very strong. The discrepancy may be due to e.g. differences in the severity of diseases, the extent that these diseases and their symptoms are considered as contributing to poor perceived health, or because some unknown direct effects of perceived health on mortality are more relevant for circulatory disease.

Confounding may not, however, be the only explanation for the mortality differentials by perceived health. The association between perceived health and accidental 
and violent mortality observed in this study (especially among men) indicates that the association may partly reflect psychosocial components of perceived health. Low sense of mastery and low sense of coherence (Lundberg and Nyström-Peck 1994), poor personal relationships, and exposure to strain potentially help us to understand the association between perceived health and accidental and violent mortality. However, current research does not allow us to explicitly assess these possibilities.

\section{Conclusions}

This study has shown that perceived health is strongly related to mortality also after sociodemographic adjustments are made. The strength of the association varies by cause of death. Attempts to understand the association between perceived health and mortality should thus begin at the cause-specific level. Medically confirmed diagnoses, life-style and risk factors as well as psychosocial determinants relevant for specific causes of death should be examined in further longitudinal studies on the association of perceived health and cause-specific mortality.

\section{Acknowledgements}

This work has been supported by the Gyllenberg Foundation and two grants from the Academy of Finland (grant 48600, 37631). We are grateful to Dr. Kristiina Manderbacka and Dr. Marja Jylhä for useful comments on an earlier draft of the paper.

\section{References}

Appels, A., H. Bosma, V. Grabauskas, A. Gostautas and F. Sturmans. 1996. Self-rated health and mortality in a Lithuanian and a Dutch population. Social Science and Medicine 42(5):681-689.

Aromaa, A., M. Heliövaara, O. Impivaara, P. Knekt, J. Maatela, M. Joukamaa, T. Klaukka, V. Lehtinen, T. Melkas, E. Mälkiä, K. Nyman, I. Paunio, A. Reunanen, K. Sievers, E. Kalimo, and V. Kallio. 1989. Health, functional limitations and need for care in Finland: Basic results from the Mini-Finland Health Survey (in Finnish with English summary). Helsinki \& Turku: Publications of the Social Insurance Institution AL:32.

Clayton, D. and M. Hills. 1993. Statistical models in epidemiology. Oxford University Press, Oxford.

Fylkesnes K. 1993. Determinants of health care utilization - visits and referrals. Scandinavian Journal of Social Medicine 21:40-50.

Heliövaara, M., A. Aromaa, T. Klaukka, P. Knekt, M. Joukamaa, and O. Impivaara. 1993. Reliability and validity of interview data on chronic diseases, the Mini-Finland Health Survey. Journal of Clinical Epidemiology 46(2):181-191. 
Hulka, B.S. and J.R. Wheat. 1985. Patterns of utilization, the patient perspective. Medical Care 23:438-460.

Idler, E.L. 1992. Self-assessed health and mortality: A review of studies. In: International review of health psychology 1 , edited by S. Maes, H. Leventhal and M. Johnston, pp. $33-54$.

Idler, E.L. and R.J. Angel. 1990. Self-rated health and mortality in the NHANES-I Epidemiologic Follow-up Study. American Journal of Public Health 80:446-452.

Idler, E.L. and Y. Benyamini. 1997. Self-rated health and mortality: A review of twentyseven community studies. Journal of Health and Social Behavior 38:21-37.

Idler, E.L., S.V. Kasl, J.H. Lemke. 1990. Self-evaluated health and mortality among the elderly in New Haven, Connecticut, and Iowa and Washington County, Iowa, 1982-1986. American Journal of Epidemiology 131:91-103.

Johnson, R.J. and F.D. Wolinsky. 1993. The structure of health status among older adults: disease, disability, functional limitation, and perceived health. Journal of Health and Social Behaviour 34(2):105-21.

Jylhä, M., E. Leskinen, E. Alanen, A-L Leskinen, and E. Heikkinen. 1986. Self-rated health and associated factors among men of different ages. Journal of Gerontology 41(6):710717.

Kaplan, G.A. and T. Camacho. 1983. Perceived health and mortality: A nine year follow-up of the Human Population Laboratory cohort. American Journal of Epidemiology 117:292304.

Kaplan G.A., D.E. Goldberg, S.A. Everson, R.D. Cohen, R. Salonen, J. Tuomilehto, and J. Salonen. 1996. Perceived health status and morbidity and mortality: Evidence from the Kuopio Ischaemic Heart Disease Risk Factor Study. International Journal of Epidemiology 25(2):259-265.

Krakau, I. 1991. Perception of health and use of health care services in a Swedish primary care district. A ten year's perspective. Scandinavian Journal of Primary Health Care 9:103-108.

Lundberg, O. and K. Manderbacka. 1996. Assessing reliability of a measure of self-rated health. Scandinavian Journal of Social Medicine 24:218-224.

Lundberg, O. and M. Nyström-Peck. 1994. Sense of coherence, social structure and health. European Journal of Public Health 4:252-257.

Lääkintöhallitus. 1969. Tautiluokitus. Valtion painatuskeskus, Helsinki.

Maddox, G.L. and E.B. Douglas. 1973. Self-assessment of health: A longitudinal study of elderly subjects. Journal of Health and Social Behavior 14:87-93.Martikainen, P., A. Aromaa, M. Heliövaara, T. Klaukka, P. Knekt, J. Maatela, and E. Lahelma. 1998. Reliability of perceived health by sex and age. Social Science \& Medicine 48:1117-1122.

Rakowski, W., V. Mor and J. Hiris. 1991. The association of self-rated health with two-year mortality in a sample of well elderly. Journal of Aging and Health 3/4:527-545.

Roos, N.P., E. Shapiro 1981. The Manitoba Longitudinal Study on Ageing: preliminary findings on health care utilization by the elderly. Medical Care 19:644-657.

Stata Reference Manual: Release 3. 1992. 5th ed. Computing Resource Center. Santa Monica, CA.

Tissue, T. 1972. Another look at self-rated health among the elderly. Journal of Gerontology 27(1):91-94. 